\title{
Impacto de la ausencia simulada de la actividad minera en México: el caso de Zacatecas
}

edgar David Gaytán Alfaro*

y AXel Arside Rodríguez Quintero**

\section{RESUMEN}

El objetivo de la presente investigación es analizar los efectos que en la economía del estado de Zacatecas tiene la hipotética extracción del subsector 212 de minería de minerales metálicos y no metálicos acorde al Sistema de Clasificación Industrial de América del Norte (SCIAN). Los resultados resaltan el impacto hacia el conjunto de la estructura productiva en Zacatecas considerando un esquema regional de insumoproducto. Se distinguen los subsectores con mayor afectación ante el escenario descrito en su perspectiva de demanda intermedia. Finalmente, se exploran alternativas sectoriales para la diversificación y fortalecimiento del mercado interno a partir de un enfoque de sostenibilidad del medio ambiente.

Palabras clave: Estudios sectoriales; minería de otros recursos no renovables; Crecimiento económico; economía de Zacatecas.

Clasificación JEL: L7; L72; O40.

Profesor-investigador de tiempo completo en el Departamento de Estudios Económicos, El Colegio de la Frontera Norte, México. Correo electrónico: davidgaytan@colef.mx

** Maestro en Economía Regional y Sectorial. Correo electrónico: axelrodriguez394@gmail.com 


\section{ABSTRACT \\ Simulated absence impact of mining activity in Mexico: the case of Zacatecas}

The purpose of this research is to analyse the hypothetical extraction effect over the 212 mining of metallic and non-metallic minerals subsector at the Zacatecas' state economy in accordance with the North American Industrial Classification System (NAICS). The results highlight the impact towards the whole productive structure in Zacatecas. They indicate a regional input-output scheme, specifically, they distinguish the subsectors with the greatest impact on the scenario described at their intermediate demand perspective. Finally, it will explore sectorial alternatives for diversification and internal market modifications based on an environmental sustainability approach.

Keywords:Sector Studies; Mining of Other Non-Renewable Resources; Economic growth; Economy of Zacatecas.

JEL Classification: L7; L72; O40.

\section{INTRODUCCIÓN}

La minería es una actividad productiva que depende totalmente de la dotación de los recursos del subsuelo de una región para su explotación (Benita et al., 2012). A nivel macro se considera como una actividad productiva generadora de ingresos; pero a nivel micro, genera externalidades negativas para la población que habita cerca de las explotaciones mineras de mediana y gran escala (Martínez, 2016).

$\mathrm{Su}$ importancia a nivel mundial radica en que es una actividad que provee de insumos a múltiples industrias, genera empleos e infraestructura, y produce efectos de derrama hacia otras actividades económicas. En México, la minería tiene gran tradición (se remonta a los tiempos precolombinos); está presente en 24 de las 32 entidades (Bancomext, 2015). La nación se ubica dentro de los 13 principales productores mundiales de 24 minerales, debido a la riqueza del subsuelo de diversos minerales de importancia internacional, entre los que destacan la plata, en primer lugar, y el oro, en séptimo. Por lo anterior, es señalada como una "actividad estratégica para el desarrollo nacional" (DOF, 2014; Servicio Geológico Mexicano, 2019). No obstante, en el país las explo- 
taciones mineras se distinguen porque no promueven la diversificación económica local y no se encuentran integradas a otras actividades de la región (García et al., 1998).

El surgimiento de la minería actual se centra en los años 1992, 1993 y 1996. En 1992 se permitió la comercialización de tierras ejidales debido a la modificación del artículo 27 constitucional. En 1993 se modificó la Ley Minera, y en 1996 se permitió que empresas extranjeras tuvieran propiedades o concesiones mineras debido a cambios en la Ley de Inversión Extranjera (Ceja, 2014).

En 2018 Zacatecas produjo $21.61 \%$ de la producción minera, lo que la ubica en el segundo lugar nacional sólo por debajo de Sonora (SGM, 2019). Esta entidad se caracteriza por un nivel de industrialización bajo, por lo que Delgado et al. (1991) aseveran que, si no fuera por la minería en la entidad, este nivel sería, inclusive, menor. Como actividad esencial, ha generado múltiples asentamientos poblacionales debido a los yacimientos de minerales y, subsecuentemente, inversión acompañada de un relativo dinamismo de la economía. A pesar de lo anterior, Benita et al. afirman que la minería es una ventaja comparativa tradicional y una "condición necesaria, pero de ninguna manera suficiente, para garantizar el desarrollo económico" (2012: 57).

Por lo anterior, el objetivo de la presente investigación es analizar los efectos que en la economía zacatecana tiene la hipotética extracción del subsector 212 de minería de minerales metálicos y no metálicos del Sistema de Clasificación Industrial de América del Norte (SCIAN). Esta región está condicionada por una configuración productiva poco diversificada y cuyos procesos históricos determinaron un patrón de especialización acendrado hacia la actividad minera. En el contexto de su particular tejido productivo, ha implicado escasos procesos de articulación sectorial y de conformación de redes de proveeduría sustentadas en procesos de acumulación de riqueza tendientes a la transmigración de actividades definidas por la agregación de valor con base en la tecnificación, la productividad y una mayor retribución a los factores de la producción.

La investigación consta de cuatro apartados. En el primero se presenta una revisión de literatura referente a la actividad minera en Zacatecas, así como de metodologías para el análisis de sectores clave mediante el uso de matrices insumo-producto. En el segundo, se explicita la metodología de extracción hipotética. En el tercero se describen 
los resultados obtenidos y discusión de ellos. Finalmente, se exponen las conclusiones y la valoración normativa del estudio.

\section{REVISIÓN DE LITERATURA}

\subsection{Minería en Zacatecas}

La actividad minera en Zacatecas se remonta a tiempos precolombinos y cobra un especial impulso durante el periodo colonial. En 1549, esta región era considerada como las de mayor importancia en el desarrollo de la minería debido al método de amalgamación que generaba mayor rendimiento de la plata, aunado a los abundantes minerales y las cuantiosas explotaciones que permitían transferir cuantiosos volúmenes a España. Así, Zacatecas se convirtió en uno de los centros económicos más importantes en la Nueva España (Burnes, 2006; Martínez, 2016; Gaytán et al., 2018).

Bakewell (1976) y Burnes (1987) explican amplia y detalladamente la minería en Zacatecas en el siglo XIX para conocer la evolución de esta actividad. Mientras que Alberro (1985) señala los ciclos económicos de largo plazo y su relación con la producción minera.

En 2012 se constituye el Clúster Minero de Zacatecas (Clusmin), el cual integra a empresas mineras de importancia como Peñoles S.A de C.V.; Minera Frisco, S.A.B. de C.V.; Fresnillo PLC S.A. de C.V.; Capstone Gold, S.A. de C.V., y Goldcorp S.A., así como instituciones académicas y de gobierno y más de 40 empresas proveedoras. Este Clúster se establece con el fin de representar compañías mineras y proveedoras, además de realizar investigación y desarrollos para mejorar la eficiencia en los procesos productivos (Solleiro et al., 2017).

La minería de oro en el estado representó $17.4 \%$ del total nacional en 2017. Peñasquito de Goldcorp fue la productora más importante de este metal en ese año. La plata, por su parte, aporta $42 \%$ del total de la producción nacional debido a las empresas Peñasquito, Saucito y Fresnillo; tan sólo la primera empresa, contribuye con $11.5 \%$ del total. En zinc también es la principal, con $48.7 \%$ de la producción mexicana gracias a las empresas Goldcorp e Industrias Peñoles. En 2017, la producción zacatecana contabilizó $13.26 \%$. Cabe destacar que ha habido una importante reducción del territorio concesionado a pesar del incremento del número de títulos. En 2005 existían 2,316 títulos que abarcaban concesiones de $1.64 \%$ de la entidad; en 2012 se dio la mayor 
cobertura, ya que $40.17 \%$ del territorio estaba concesionado a 2,618 títulos. En 2019 disminuyó drásticamente a $0.02 \%$ de la entidad para 2,671 títulos (Camimex, 2018; SGM, 2018).

En años recientes, la explotación de los yacimientos minerales lo realizan en su mayoría empresas extranjeras. Esto ocasiona una menor articulación entre los sectores económicos al interior de la entidad dado que ni invierten ni demandan ni ofertan insumos en Zacatecas. Aunado a ello, la economía estatal no ha realizado cambios importantes en su perfil productivo debido a que el gobierno estatal no han generado las condiciones favorables o políticas requeridas para lograr dichos cambios (Gaytán et al., 2017).

\subsection{Análisis de sectores clave: extracción hipotética}

Conocer los sectores productivos de la actividad económica es esencial para identificar aquellos que dinamicen e impulsen una economía, así como planificar y generar políticas que propicien crecimiento económico y desarrollo regional. Esto favorecerá la mejora de indicadores macroeconómicos y microeconómicos (Cardenete y Delgado, 2011; Temurshoev, 2009; Sancho y Cardenete, 2014).

La detección de los sectores clave se puede obtener por múltiples metodologías. Por ejemplo, para Hirschman (1958; citado por Viladecans, 1999), pueden identificarse a partir de un razonamiento de encadenamientos que pueden ir hacia atrás (backward linkages), cuando un sector demanda insumos de otros, lo que genera efectos de producción en otras industrias. Y los encadenamientos hacia adelante (forward linkages), cuando un sector provee de insumos a otros para continuar con el proceso de producción.

Rasmussen (1956; citado por Sancho y Cardenete, 2014) señala que es posible detectar los sectores por medio del poder de dispersión y de absorción económica. El primero ofrece insumos a otras áreas, y el segundo, al análisis de la afectación de un sector cuando el resto incrementa una unidad su demanda final. Los ramos clave se obtienen por cálculos a partir de la Matriz Inversa de Leontief.

Al respecto, Chenery y Watanabe (1958) señalan que los encadenamientos hacia atrás cuantifican la capacidad de un sector de arrastrar al resto por la demanda bienes intermedios, por lo que estimula la actividad de las otras áreas. Éste se calcula como una proporción de las 
compras intermedias al sector entre el total de su producción efectiva (Moncaleano, 2015).

Por su parte, los Coeficientes de Streit cuantifican tanto los encadenamientos hacia atrás y hacia adelante como los vínculos sectoriales directos e indirectos. Se calcula como la media aritmética de los cuatro encadenamientos posibles entre dos sectores, lo que genera una matriz de efectos multiplicadores (Moncaleano, 2015).

\section{Metodología}

De manera cronológica, la primera propuesta de la metodología de extracción hipotética (HEM, por sus siglas en inglés), fue la Paelinck et al. (1965), que fue mejorada en trabajos realizados por Strassert (1968), Schultz (1977), Cella (1984), Clements (1990) y Heimler (1991). Asimismo, son destacables las investigaciones de Miller \& Lahr (2001), así como Cai y Leung (2004). En la presente investigación se aplica la metodología de Dietzenbacher et al. (1993) ${ }^{1}$.

El HEM permite cuantificar la importancia de uno o varios sectores por su relevancia económica tras la hipotética extracción de un área. La producción reducida debido a la eliminación del sector cuantifica los vínculos que se generan, así como las dependencias con las que el ramo cuenta. De esta manera es posible determinar los ámbitos con mayor importancia en una economía (Guerra, 2011; Andreosso-O'Callaghan y Yue, 2004).

La metodología de extracción hipotética permite analizar los efectos que tendrían el resto de los sectores a partir de su eliminación del modelo insumo-producto, particularmente en la submatriz de demanda intermedia. La extracción implica que el ramo no vende ni compra ningún insumo intermedio a sí mismo u a otros; es decir, se supone que las importaciones satisfacen la oferta y demanda del sector extraído. Este método busca cuantificar los eslabonamientos para el resto de las áreas que generan la extracción o eliminación de un sector. Se calcula la diferencia entre el output total alcanzado a partir del total de los sectores y el "output disminuido", que se obtiene por la eliminación de algún

\footnotetext{
Se utilizó el lenguaje Python 3.6 empleando la propuesta operativa de Nazara et al. (2003), en el marco de los trabajos del Laboratorio de Economía Aplicada de la Universidad de Illinois y el Banco de la Reserva Federal en Chicago, para el desarrollo metodológico.
} 
área específica, para cuantificar la importancia del sector extraído. Esta metodología se utiliza principalmente para detectar ramos clave (Yuhuan et al., 2016; Llano, 2009; Yu et al., 2006).

El HEM genera dos efectos: el de arrastre y el de difusión, es decir, hacia atrás y hacia adelante, respectivamente. En el primero se hace uso de la Matriz Inversa de Leontief; en el segundo se utiliza la matriz Ghoshiana $^{2}$ (Cansino et al., 2013). En esta investigación se busca cuantificar el valor bruto de la producción (VBP) tras la eliminación de los sectores, tanto hacia atrás como hacia adelante.

El VBP tras la eliminación de un sector se cuantifica mediante la Matriz Inversa de Leontief. Esto se expresa como:

$$
x-\bar{x}=\left(\begin{array}{l}
x^{1}-\bar{x}^{1} \\
x^{R}-\bar{x}^{R}
\end{array}\right)=\left\{\left[\begin{array}{ll}
L^{11} & L^{1 R} \\
L^{R 1} & L^{R R}
\end{array}\right]-\left[\begin{array}{cc}
\left(I-A^{11}\right)^{-1} & 0 \\
0 & \left(I-A^{R R}\right)^{-1}
\end{array}\right]\right\}\left(\begin{array}{l}
f^{1} \\
f^{R}
\end{array}\right)
$$

Donde $x$ es el VBP total del sistema con todos los sectores, $x$ el VBP extraído el sector de estudio, $L$ la Matriz Inversa de Leontief, $A$ la matriz de coeficientes técnicos, $f$ el vector de demanda final, los supraíndices $j$ y $r$ señalan el sector eliminado y el resto de los sectores económicos, respectivamente; y el subíndice $n$, el orden de las matrices que coincidirá con el de los sectores productivos de la matriz insumo-producto.

Para el segundo efecto, el de difusión o hacia adelante, se utiliza la Matriz Inversa Ghoshiana $(G)$, el vector de insumos primarios $(v)$ y la matriz de coeficientes de distribución $(B)$. Es decir:

$$
x-\bar{x}=\left(v^{i^{i}}-v^{r^{\prime}}\right)=\left\{\left[\begin{array}{ll}
G^{11} & G^{1 R} \\
G^{R 1} & G^{R R}
\end{array}\right]-\left[\begin{array}{cc}
\left(I-B^{11}\right)^{-1} & 0 \\
0 & \left(I-B^{R R}\right)^{-1}
\end{array}\right]\right\}
$$

La reducción del VBP tras la extracción de los subsectores no solamente es por la formación de riqueza, sino también por el intercambio de insumos en el aparato productivo, en donde existen interdependencias sectoriales que generan una serie de afectaciones.

2 El uso de la matriz Ghoshiana en la metodología de extracción hipotética resulta del trabajo de Ghosh (1958). Se obtiene del impacto en la estructura de insumos ocasionado por modificaciones en los costos cuantificados en la composición de valor agregado de cada sector. 
Los cálculos se realizaron a partir de la matriz insumo-producto regional de Zacatecas, a una desagregación de 56 subsectores según el Sistema de Clasificación Industrial para América del Norte. Aunque ésta es de 79 subsectores, solamente se hizo uso de aquellos que tienen información de los Censos Económicos 2014, esto es, 56 subsectores de información. De tal desagregación de la matriz, el subsector de mayor interés es el 212 de Minería de minerales metálicos y no metálicos, excepto petróleo y gas, debido a su importancia en la actividad económica zacatecana.

\section{RESULTADOS}

Se hizo uso de la Matriz de Insumo Producto de la Economía de Zacatecas cuantificada en millones de pesos a precios básicos de 2013 (MIPZac-13), para la obtención de los resultados. Lo anterior se define como un proceso de regionalización de Insumo Producto que fue llevado a cabo por recursos no survey, siguiendo la metodología propuesta por Flegg y Webber (1997), la cual, en primera instancia, requiere el cálculo del coeficiente con el mismo nombre de los autores:

$$
F L Q_{i j}=\left(C I L Q_{i j}\right)\left(\lambda_{r}^{\delta}\right)\left(a_{i j}\right)
$$

Donde:

FLQij= Coeficiente de Flegg-Webber;

$C I L Q i j=$ Coeficientes de localización de industria cruzada;

$\lambda_{\mathrm{r}}^{\delta}=$ Factor de ponderación del tamaño relativo de la región;

$a i j=$ Coeficientes técnicos nacionales de insumo producto.

De la ecuación 3 se desprende $\lambda_{\mathrm{r}}^{\delta}$ como un elemento clave, debido a que ilustra un ponderador del tamaño relativo de la región contenido en $\delta$, que puede ser igual o mayor a la unidad. Basados en la evidencia empírica, para Flegg y Webber fijar un logaritmo base dos representa una ponderación promedio del tamaño relativo de la región subnacional; cualquier otra discrepa de la media, hecho que indica autosuficiencia de 
la región, o bien una subrepresentación de los territorios subnacionales.

De manera que:

$$
\lambda_{r}^{\delta}=\log _{2}\left(1+Y_{r} / Y_{n}\right)^{\delta}
$$

Donde:

$Y_{r}=$ Valor agregado bruto regional;

$Y_{\mathrm{r}}=$ Valor agregado bruto nacional.

\subsection{Backward Linkage}

Los resultados obtenidos de los Backward Linkage se muestran en el Cuadro 1. Se aprecia que el subsector 431 de Comercio es el que genera mayor impacto en la formación de VBP, porque se perdería $19.71 \%$, lo que es superior a $2.88 \%$ generado en la MIP-ZAC-13. Posteriormente, se puede observar que los subsectores relativos a las manufacturas también impactarían de manera importante al VBP, debido a la gran demanda de insumos que requieren para realizar sus actividades productivas. Sin embargo, el subsector 312 de Industria de las bebidas y del tabaco muestra que la aportación al VBP (19.37\%) es mayor al impacto que generaría tras su hipotética extracción (4.12\%), lo cual puede explicarse dado que requieren de importantes insumos para la producción de la planta cervecera en los que se ha invertido, pero que, por su prescindencia, se destinarían en menor cantidad a otros subsectores.

En cuanto al subsector 212 de Minería de minerales metálicos y no metálicos, excepto petróleo y gas, objeto de estudio de esta investigación, se muestra que generaría un impacto considerablemente menor a lo que actualmente aporta al VBP, lo cual puede deberse a que requiere de múltiples insumos para realizar las actividades que genera; no obstante, ante la eliminación del subsector, los insumos serían utilizados en menor medida en otros. 
Cuadro 1

IMPORTANCIA DE SUBSECTORES DE LA MIP-ZAC-13 POR VBP ANTE LA APLICACIÓN DE LA HEM BACKWARD LINKAGE (MILLONES DE PESOS A PRECIOS BÁSICOS)

\begin{tabular}{|c|c|c|c|c|}
\hline No. & Subsector & VBP Perdido* & $\begin{array}{l}\% \text { Aportación al } \\
\text { VBP MIP-Zac-13** }\end{array}$ & $\begin{array}{l}\text { Impacto } \\
\text { HEM }^{* * *}\end{array}$ \\
\hline 431 & Comercio & $1,084.85$ & 2.88 & 19.72 \\
\hline 336 & Fabricación de equipo de transporte & $1,014.42$ & 12.35 & 18.44 \\
\hline 311 & Industria alimentaria & 329.66 & 2.46 & 5.99 \\
\hline 327 & $\begin{array}{l}\text { Fabricación de productos a base de minerales no } \\
\text { metálicos }\end{array}$ & 328.65 & 1.20 & 5.97 \\
\hline 332 & Fabricación de productos metálicos & 319.55 & 1.64 & 5.81 \\
\hline 212 & $\begin{array}{l}\text { Minería de minerales metálicos y no metálicos, } \\
\text { excepto petróleo y gas }\end{array}$ & 310.13 & 44.96 & 5.64 \\
\hline 326 & Industria del plástico y del hule & 226.87 & 0.97 & 4.12 \\
\hline 312 & Industria de las bebidas y del tabaco & 226.71 & 19.37 & 4.12 \\
\hline 561 & Servicios de apoyo a los negocios & 207.49 & 0.98 & 3.77 \\
\hline 522 & $\begin{array}{l}\text { Instituciones de intermediación crediticia y } \\
\text { financiera no bursátil }\end{array}$ & 133.53 & 1.65 & 2.43 \\
\hline
\end{tabular}

* Monto del Valor Bruto de la Producción que se perdería en el aparato del conjunto productivo ante la ausencia simulada del subsector respectivo.

** Porcentaje de aportación a la formación del VBP.

*** Porcentaje de reducción en el VBP como medida de impacto en el conjunto de la economía.

Fuente: elaboración propia con los resultados de la Metodología de Extracción Hipotética de la MIPZac2013.

\subsection{Forward Linkage}

Se cuantifica la importancia de los subsectores debido a su participación en la demanda intermedia como proveedores de insumos y de procesos de generación de valor; es decir, encadenamientos hacia adelante. La extracción de un sector implicaría la imposibilidad de una economía de contar y proveer de los insumos requeridos para el proceso de producción. Los resultados se muestran en el Cuadro 2.

El subsector 431 de Comercio es el que mayor impacto generaría en el VBP tras su hipotética extracción, ya que disminuiría $20.63 \%$, en comparación con $2.88 \%$ que genera en la MIP-Zac-13. Es decir, no genera VBP, pero es un importante proveedor de insumos. Se muestran diversos subsectores referentes a las manufacturas que impactarían más tras su eliminación que lo que generan en la MIP-Zac-13, debido a que 
son productores de insumos a otros subsectores. Por su parte, el impacto tras la eliminación del subsector 312 de Industria de las bebidas y del tabaco sería menor que el que genera.

Respecto al subsector 212 de Minería de minerales metálicos y no metálicos, excepto petróleo y gas, ocasionaría un impacto de 5.91\% del VBP, lo que es menor al $44.95 \%$ que genera. Esto hace referencia a que no es proveedor de insumos a otros subsectores, ya sea para su transformación y agregación de valor o para la comercialización, sino que es, predominantemente, una actividad extractiva.

\section{Cuadro 2}

IMPORTANCIA DE SUBSECTORES DE LA MIP-ZAC-13 POR VBP ANTE LA APLICACIÓN DE LA HEM FORWARD LINKAGE (MILLONES DE PESOS A PRECIOS BÁSICOS)

\begin{tabular}{|c|c|c|c|c|}
\hline No. & Subsector & VBP Perdido* & $\begin{array}{l}\text { \% Aportación al } \\
\text { VBP MIPZac13** }\end{array}$ & $\begin{array}{l}\text { Impacto } \\
\text { HEM }^{* * *}\end{array}$ \\
\hline 431 & Comercio & $1,149.05$ & 2.88 & 20.63 \\
\hline 336 & Fabricación de equipo de transporte & $1,004.41$ & 12.35 & 18.04 \\
\hline 327 & $\begin{array}{l}\text { Fabricación de productos a base de minerales no } \\
\text { metálicos }\end{array}$ & 329.22 & 1.20 & 5.91 \\
\hline 212 & $\begin{array}{l}\text { Minería de minerales metálicos y no metálicos, } \\
\text { excepto petróleo y gas }\end{array}$ & 329.19 & 44.96 & 5.91 \\
\hline 332 & Fabricación de productos metálicos & 321.81 & 1.64 & 5.78 \\
\hline 311 & Industria alimentaria & 320.54 & 2.46 & 5.76 \\
\hline 326 & Industria del plástico y del hule & 230.26 & 0.97 & 4.13 \\
\hline 561 & Servicios de apoyo a los negocios & 219.13 & 0.98 & 3.93 \\
\hline 312 & Industria de las bebidas y del tabaco & 214.56 & 19.37 & 3.85 \\
\hline 522 & $\begin{array}{l}\text { Instituciones de intermediación crediticia y } \\
\text { financiera no bursátil }\end{array}$ & 130.10 & 1.65 & 2.34 \\
\hline
\end{tabular}

* Monto del Valor Bruto de la Producción que se perdería en el aparato del conjunto productivo ante la ausencia simulada del subsector respectivo.

** Porcentaje de aportación a la formación del VBP.

*** Porcentaje de reducción en el VBP como medida de impacto en el conjunto de la economía.

Fuente: elaboración propia con los resultados de la Metodología de Extracción Hipotética de la MIPZac2013.

Al sujeto de estudio, el subsector 212 de Minería de minerales metálicos y no metálicos, excepto petróleo y gas, muestra que, tanto en el backward como en el forward linkage, sería uno de los principales cuya 
prescindencia tendría un impacto fuerte, a pesar de que la aportación del VBP es muy alta. A continuación se señala las implicaciones que este sector generaría específicamente a otros subsectores.

\subsection{Impacto específico en la minería zacatecana}

En este apartado se muestran los resultados específicos del subsector 212 sobre el aparato productivo de la economía zacatecana, como demandante y como proveedor de insumos. Esto se obtiene a partir de la ecuación (5):

$$
\mu(k)=\frac{\sum_{i=1, i \neq k}^{79}\left(V B P_{i}-\overline{V B P}_{i}(k)\right)}{\sum_{i=1}^{79} \sum_{i=1, i \neq k}^{79}\left(V B P_{i}-\overline{V B P}_{i}(k)\right)} \cdot(100)=\frac{L(k)}{\sum_{i=1}^{79} L(k)} \cdot(100)
$$

Donde $\overline{V B P}(k)$ representa el valor bruto de la producción tras la modificación en la omisión del k-ésimo sector, y $\mu(k)$ denota el impacto relativo total de la ausencia del sector k-ésimo, tanto si el enfoque es hacia atrás como hacia delante.

Se cumpliría la ecuación anterior, si:

$$
\mu_{i}(k)=\frac{l_{i}(k)}{\sum_{i=1}^{79} L(k)} *(100)
$$

Donde $l_{i}(k)$ denota el efecto en el sector i-ésimo en el VBP que se opera a consecuencia de la ausencia del sector k-ésimo. Por lo anterior, es cierto que $\mu(k)=\sum \mu_{i}(k)$.

Los impactos específicos correspondientes al backward linkages del subsector 212 se muestran en el Cuadro 3. De manera desagregada, se se expone el porcentaje específico de afectación a cada subsector de $5.63 \%$ de impacto tras su hipotética extracción. El mayor monto de reducción de VBP es del mismo subsector, debido a que impactaría en 267,796 millones de pesos y $4.87 \%$ en valor relativo. Por ello, el impacto al resto del aparato productivo desagregado en los otros 55 subsectores es muy reducido. 
Otros subsectores que también enfrentarían afectaciones importantes son los servicios del 431 de Comercio y 561 de Servicios de apoyo a los negocios, así como las industrias 238 de Trabajos especializados en construcción, y 811 de Servicios de reparación y mantenimiento, entre otros. El comercio es el segundo subsector con mayor impacto con $0.23 \%$ de la afectación del subsector de interés. Este último, con los restantes 8 subsectores del tabulador, suman $0.47 \%$ de la hipotética contracción en el VBP.

\section{Cuadro 3}

EFECTO DE LA EXTRACCIÓN DEL SUBSECTOR 212 SOBRE EL CONJUNTO DE LA ECONOMÍA, BACKWARD LINKAG (MILLONES DE PESOS A PRECIOS BÁSICOS)

\begin{tabular}{|c|c|c|c|c|}
\hline No. & Subsector & VBP Perdido* & $\begin{array}{l}\text { Aportación al } \\
\text { VBP perdido** }\end{array}$ & $\begin{array}{c}\text { Impacto } \\
\text { desagregado*** }\end{array}$ \\
\hline 212 & $\begin{array}{l}\text { Minería de minerales metálicos y no metálicos, } \\
\text { excepto petróleo y gas }\end{array}$ & 267.80 & 86.35 & 4.87 \\
\hline 431 & Comercio & 12.62 & 4.07 & 0.23 \\
\hline 561 & Servicios de apoyo a los negocios & 6.11 & 1.97 & 0.11 \\
\hline 238 & Trabajos especializados para la construcción & 4.60 & 1.48 & 0.08 \\
\hline 811 & Servicios de reparación y mantenimiento & 3.90 & 1.26 & 0.07 \\
\hline 332 & Fabricación de productos metálicos & 3.29 & 1.06 & 0.06 \\
\hline 336 & Fabricación de equipo de transporte & 3.19 & 1.03 & 0.06 \\
\hline 541 & Servicios profesionales, científicos y técnicos & 2.87 & 0.92 & 0.05 \\
\hline 484 & Autotransporte de carga & 1.24 & 0.40 & 0.02 \\
\hline 321 & Industria de la madera & 0.92 & 0.30 & 0.02 \\
\hline
\end{tabular}

* Monto de VBP perdido a consecuencia de la omisión del subsector 212. Valoraciòn por Backward Linkage, millones de pesos a precios básicos.

** Porcentaje de contribución al total del VBP perdido por subsector como consecuencia de la extracción del subsector 212.

*** Contribución relativa al 5.64\% de reducción en el VBP total en la economía zacatecana que resulta de la extracción del subsector 212.

Fuente: elaboración propia con los resultados de la Metodología de Extracción Hipotética de la MIPZac2013.

Los resultados del forward linkages de la extracción del subsector 212 se muestran en el Cuadro 4.

El mayor impacto se presenta en el subsector 327 de Fabricación de productos a base de minerales no metálicos, con $3.23 \%$ del impacto 
total del subsector extraído. Este resultado se debe aque es una actividad con estrecha relación con el subsector extraído, debido a su importancia en el proceso de agregación de valor de los insumos obtenidos de la minería.

El segundo subsector con mayor impacto es el de estudio, con $0.73 \%$ de afectación de $5.91 \%$. Esto afirma que la proveeduría de insumos impactaría a otros subsectores más que a sí mismo debido a su carácter eminentemente primario y extractivo. Los resultados señalan que esta acción se diluye principalmente en subsectores asociados a las industrias. Los nueve subsectores posteriores del 327 suman 2.61\%, por lo que el impacto es mayor hacia adelante que hacia atrás.

\section{Cuadro 4}

EFECTO DE LA EXTRACCIÓN DEL SUBSECTOR 212 SOBRE EL CONJUNTO DE LA ECONOMíA, FORWARD LINKAGE (MILLONES DE PESOS A PRECIOS BÁSICOS)

\begin{tabular}{|c|c|c|c|c|}
\hline No. & Subsector & VBP Perdido* & $\begin{array}{l}\text { Aportación al } \\
\text { VBP perdido** }\end{array}$ & $\begin{array}{c}\text { Impacto } \\
\text { desagregado*** }\end{array}$ \\
\hline 327 & $\begin{array}{l}\text { Fabricación de productos a base de minerales } \\
\text { no metálicos }\end{array}$ & 180.15 & 54.72 & 3.23 \\
\hline 212 & $\begin{array}{l}\text { Minería de minerales metálicos y no metálicos, } \\
\text { excepto petróleo y gas }\end{array}$ & 40.90 & 12.42 & 0.73 \\
\hline 331 & Industrias metálicas básicas & 32.54 & 9.88 & 0.58 \\
\hline 312 & Industria de las bebidas y del tabaco & 28.18 & 8.56 & 0.51 \\
\hline 237 & Construcción de obras de ingeniería civil & 14.70 & 4.47 & 0.26 \\
\hline 336 & Fabricación de equipo de transporte & 11.97 & 3.63 & 0.21 \\
\hline 236 & Edificación & 9.76 & 2.97 & 0.18 \\
\hline 311 & Industria alimentaria & 3.27 & 0.99 & 0.06 \\
\hline 332 & Fabricación de productos metálicos & 2.67 & 0.81 & 0.05 \\
\hline 326 & Industria del plástico y del hule & 1.62 & 0.49 & 0.03 \\
\hline
\end{tabular}

* Monto de VBP perdido a consecuencia de la omisión del subsector 212. Valoraciòn por Forward Linkage, millones de pesos a precios básicos.

** Porcentaje de contribución al total del VBP perdido por subsector como consecuencia de la extracción del subsector 212.

*** Contribución relativa al 5.91\% de reducción en el VBP total en la economía zacatecana que resulta de la extracción del subsector 212.

Fuente: elaboración propia con los resultados de la Metodología de Extracción Hipotética de la MIPZac2013. 


\subsection{Discusión}

A partir de los resultados de la metodología de Extracción Hipotética (véase cuadros 1-4) se pueden puntualizar los efectos que genera la hipotética extracción del subsector 212 de Minería de minerales metálicos y no metálicos, excepto petróleo y gas.

El análisis de los resultados puede realizarse en backward linkages (encadenamientos hacia atrás) y forward linkages (encadenamientos hacia adelante). En el primero se muestra que el subsector demanda insumos del resto, por lo que puede dinamizar el desempeño de una cadena de proveeduría. En el caso del subsector minería, objeto de estudio de esta investigación, se observa que los efectos de demanda de insumos ante su ausencia son particularmente al subsector Comercio (431), así como al de Fabricación de productos a base de minerales no metálicos (327), y al de Fabricación de productos metálicos (332); por lo que éstos proveen importantes insumos al subsector 212. En el impacto específico que el subsector genera al resto del aparato productivo, la afectación es de $86 \%$ del impacto al mismo subsector y el resto desagregado en el conjunto de las actividades económicas.

Por su parte, los forward linkages señalan los insumos que el subsector de la minería provee al resto de la economía para sus procesos productivos. Los resultados muestran que los subsectores de Comercio (431), Fabricación de equipos de transporte (336), Fabricación de productos a base de minerales no metálicos (327), Minería de minerales metálicos y no metálicos, excepto petróleo y gas (212) y Fabricación de productos metálicos (332) son los de mayor impacto, por lo que no obtendrían insumos minerales para su proceso de transformación y agregación de valor. Las actividades con mayor impacto debido a la hipotética extracción del subsector 212 son: Fabricación de productos a base de minerales no metálicos (327), Minería de minerales metálicos y no metálicos, excepto petróleo y gas (212) e Industrias metálicas básicas (331).

Asimismo, fue posible observar que las actividades con mayor impacto, tanto en los backward como en los forward linkgages, son subsectores de importancia en la economía zacatecana como la Industria alimentaria (311), la Industria de las bebidas y del tabaco (312) y Fabricación de equipo de transporte (336), por lo que la afectación de un subsector impactaría de manera significativa al resto del aparato productivo del estado. 


\section{CONCLUSIONES}

El objetivo de este trabajo fue mostrar, de forma holística, el impacto de la ausencia simulada de la actividad minera en el estado de Zacatecas, entidad cuyo perfil económico-productivo está históricamente determinado por la notable presencia de la minería. La naturaleza eminentemente extractiva de esta actividad, así como su orientación al mercado externo, son factores que sugieren escasos efectos de integración a la economía local, así como de articulación con el conjunto de los sectores de actividad económica comprendidos en el aparato productivo del estado. Los resultados son congruentes con tal afirmación, pues hay una notable aportación del subsector 212 de Minería de minerales metálicos y no metálicos a la formación del valor bruto de la producción, pero relativamente escaso impacto en la formación de dicha variable tras su hipotética exclusión, tanto en los efectos de encadenamiento hacia atrás como hacia delante. Esto es congruente con la visión de una economía altamente especializada y con pocos efectos de integración y cohesión de su aparato productivo sustetado en el tamaño y variedad de las redes de intercambio y la intensidad de los patrones de comercialización dentro de cada una de ellas.

Por último, los resultados aquí presentados pretenden ser un referente de los impactos específicos que genera la minería en Zacatecas, lo que la coloca en la dimensión de la promoción vigente del fortalecimiento del tejido productivo local con un enfoque de diversificación de la actividad económica y, por ello, de la ampliación del mercado de demanda intermedia. Lo anterior adquiere fundamento en la necesidad de trascender la condición monoproductiva del estado para lograr una dinámica de crecimiento sostenida en el largo plazo en un marco sustentable. Lograr lo anterior supone visualizar los sectores de potencial diversificación sobre una base productiva presente definida por las limitadas alternativas que ofrece el actual patrón de especialización. 


\section{Bibliografía}

Alberro, Solange (1985), "Zacatecas, zona frontera, según los documentos inquisitoriales, siglos XVI y XVII”, Estudios de historia novohispana 8(8), pp. 139-170.

Andreosso-O'Callaghan, B. y G. Yue (2004), “Intersectoral linkages and key sectors in China, 1987-1997”, Asian Economic Journal, 18 (2), pp. 165-83

Bakewell, P.J. (1976), Minería y Sociedad en el México colonial. Zacatecas 1546-1700, Fondo de Cultura Económica, México.

Bancomext (2015), "Macro coyuntura minería. Primer informe 2015”, Comercio Exterior, Dirección de Estudios Económicos, México.

Benita Maldonado, Francisco J.; Edgar D. Gaytán Alfaro y Mayra C. Rodallegas Portillo (2012), "Un estudio no paramétrico de eficiencia para la. minería de Zacatecas, México", Revista de Métodos Cuantitativos para la Economía y la Empresa, 14, pp. 54-75.

Burnes, A. (2006), El drama de la minería mexicana: del pacto colonial a la globalización contemporánea, Universidad Autónoma de Zacatecas, México.

Burnes, A. (1987), La minería en la historia económica de Zacatecas (15461876). Universidad Autónoma de Zacatecas, México.

Cai, J. y P. Leung, (2004), "Linkage measures: A revisit and a suggested alternative", Economic Systems Research, 16 (1), pp. 65-85.

Camimex (Cámara Minera de México) (2018), “Informe Anual 2018 Cámara Minera de México LXXXI Asamblea General Ordinaria”, México. $<$ https:/camimex.org.mx/files/1015/3073/8596/Info2018.pdf>

Cansino Muñoz-Repiso José Manuel; Manuel Alejandro Cardenete Flores, Manuel Ordóñez Ríos y Rocía Román Collado (2013), “Análisis de sectores clave de la economía española a partir de la Matriz de Contabilidad Social de España 2007”, Estudios de Economía Aplicada. 31(2), pp. 621653.

Cardenete Flores, Manuel Alejandro y Jorge Manuel López Álvarez (2015), "Análisis de sectores clave a través de Matrices de Contabilidad Social: El caso de Andalucía”. Estudios de Economía Aplicada. 33(1), pp. 203-222.

Ceja, Jorge (2014), "Extractivismo minero, globalización neoliberal y resistencia socio-ambiental en México", Contextualizaciones latinoamericanas 6(11), pp. 1-10.

Cella, G. (1984), "The input-output measurement of interindustry linkages", Oxford Bulletin of Economics and Statistics. 46 (1), pp. 73-84.

Chenery, H. y T. Watanabe (1958), “An International Comparison of the Structure of Production”. Econometrica, vol. 6(1), 487-521.

Clements, B.J. (1990), "On the Decomposition and Normalization of Interindustry Linkages”, Economics Letters. 33, pp. 337-340. 
Delgado Wise, Raúl; Víctor M. Figueroa y Margarita Hoffner Long (1991), Zacatecas: sociedad, economía, política y cultura, CIIIH-UNAM, México.

Dietzenbacher, E.; J.A. Van Der Linden y A. Steenge (1993), "The Regional Extraction Method: EC Input-Output Comparisons", Economic Systems Research. 5 (1), pp. 185-206.

DOF (Diario Oficial de la Federación) (2014), "ACUERDO por el que se aprueba el Programa de Desarrollo Minero 2013-2018”, Gobierno de México. <https://www.dof.gob.mx/nota_detalle.php?codigo $=5344070 \& \mathrm{fec}$ ha $=09 / 05 / 2014>$

García, M.; S. González, A. Sánchez y B. Verduzco (1998), Descentralización e iniciativas locales de desarrollo, Universidad de Guadalajara / UCLA / Programación México-Juan Pablos, México.

Gaytán Alfaro, Edgar David; Rigoberto Jiménez Díaz y Aldo Alejandro Pérez Escatel (2017), Matriz de insumo-producto para la economía del estado de Zacatecas: un enfoque de clústers, Colofón, México.

Gaytán Alfaro, Edgar David; Mario Alberto Mendoza Sánchez y Juan Roberto Vargas Sánchez (2018), "Minería y encadenamientos productivos en México: un estudio comparativo empleando modelos estatales de insumo producto", Economía coyuntural, Revista de temas de coyuntura y perspectivas 3(2), pp. 1-38.

Ghosh, A. (1958), "Input-Output Approach in Allocation System", Economica, núm. 25. pp. 58-64.

Guerra, Ana-Isabel (2011), "Merging the Hypothetical Extraction Method and the Classical Multiplier Approach: A Hybrid Possibility for Identifying Key Distributive Sectors", UFAE and IAE Working Papers, pp. 1-31.

Heimler, A. (1991), "Linkages and Vertical Integration in the Chinese Economy", The Review of Economics and Statistics. 73 (2), pp. 261-267.

Hirschman, A. (1958), The Strategy of Economic Development, Oxford University Press, EUA.

Instituto Nacional de Estadística y Geografía (INEGI) (2013), Sistema de Clasificación Industrial de América del Norte, SCIAN, México. $\quad<\mathrm{http}$ ://internet.contenidos.inegi.org.mx/contenidos/Productos/ prod_serv/contenidos/espanol/bvinegi/productos/clasificadores/SCIAN/ SCIAN_ 2013/702825051693.pdf>

Llano, Carlos (2009), "Efectos de desbordamiento interregional en España: Una estimación a través del modelo input-output interregional". Investigaciones Regionales. (16), pp. 181-188.

Martínez Barragán, Hirineo (2016), "Concesiones, explotación minera y conflicto en la frontera Jalisco-Colima", Espiral, XXIII(67), pp. 45-90. $<$ https://www.redalyc.org/articulo.oa?id=138/13846352002>

Miller, R.E. y (2001), “A taxonomy of extractions”, en M.L. Lahr y R.E. Miller (eds.), Regional Science Perspectives in Economic Analysis: A Festschrift in Memory of Benjamin H. Stevens. Elsevier Science, Amsterdam, 
pp. 407-441, .

Moncaleano Cuéllar, Lina María (2015), “Análisis de encadenamientos sectoriales y proyecciones de la demanda final que permitan plantear escenarios para mejorar la producción y reducir el desempleo en Colombia", Tesis de maestría, Facultad de Ciencias Económicas y Administrativas, Pontificia Universidad Javeriana, Colombia.

Nazara, S., D. Guo, G.J. Hewings y C. Dridi (2003), "PyIO: input-output analysis with Python”, Working Paper No. 0409002, University Library of Munich, Alemania.

Paelinck, J., J. De Caevel y J. Degueldre (1965), “Analyse quantitative de certaines phenomenes du developpment regional polarise: Essai de simulation statique d'iteraires de propogation", Bibliothequede l'Institut de Science Economique 7: Problemesde conversion economique: analyses theoretiques et etudes appliquees, M.-Th. Genin, París, pp. 341-87.

Rasmussen, P. (1956), Studies in Intersectoral Relations, North Holland, Amsterdam.

Sancho, Ferran y Manuel Alejandro Cardenete (2014), "Instrumentos multisectoriales para la detección de sectores clave en el análisis regional", $R e$ vista de Estudios Regionales. (100), pp. 131-146.

Schultz, S. (1976), "Intersectoral comparisons as an approach to the identification of key sectors", en K.R. Polenske y J.V. Skolka (eds.), Advances in Input-Output Analysis, Ballinger Publishing Company. Cambridge, Massachusetts, EUA, pp. 137-59.

Schultz, S. (1977), “Approaches to Identifying Key Sectors Empirically by Means of Input-Output Analysis”, Journal of Development Studies. 14, pp. 77-96.

SGM (Servicio Geológico Mexicano) (2018). "Panorama Minero del Estado de Zacatecas", México. <http://www.sgm.gob.mx/pdfs/ZACATECAS. pdf $>$

SGM (2019), “Anuario estadístico de la minería mexicana, 2018. Edición 2019”, 48, Pachuca, México. <http://www.sgm.gob.mx/productos/pdf/ Anuario_2018_Edicion_2019.pdf $>$

Solleiro Rebolledo, José Luis, Araceli Olivia Mejía Chávez y Brenda Susana Figueroa Ramírez (2017), "Prospectiva de la minería en el clúster de Zacatecas y los retos para la formación de capital humano", Gestión de la innovación para la competitividad: Sectores estratégicos, tecnologías emergentes y emprendimientos, ALTEC 2017 VXII Congreso LatinoIberoamericano de Gestión Tecnológica, pp. 1-18. <http:/www.uam.mx/ altec2017/pdfs/ALTEC_2017_paper_215.pdf>

Strassert, G. (1968), "Zur Bestimmung Strategischer Sektoren mit Hilfe von Input-Output Modelen", Jahrbücher für Nationalökonomie und Statistik, vol. 182, pp. 211-215.

Temurshoev, Umed (2009), "Hypothetical extraction and fields of influence 
approaches: integration and policy implications", EERC Working Paper Series, Rusia, pp. 1-42.

Viladecans Marsal, Elisabet (1999), "El papel de las economías de aglomeración en la localización de las actividades industriales. Un análisis del caso español", Tesis doctoral, Universitad de Barcelona, España.

Yu Song, Chunlu Liu y Craig Langston (2006), "Linkage measures of the construction sector using the hypotetical extraction method", Construction Management and Economics. 24(6), pp. 579-589. doi: 10.1080/01446190500435358

Yuhuan Zhao, Ya Liu, Song Wang, Zhonghua Zhang y Jiechao Li (2016), "Inter-regional linkage analysis of industrial CO2 emissions in China: An application of a hypothetical extraction method", Ecological Indicators, 61, pp. 428-437. doi: 10.1016/j.ecolind.2015.09.044

\section{ANEXO}

Cuadro A.1

56 SUBSECTORES UTILIZADOS DEL SISTEMA DE CLASIFICACIÓN INDUSTRIAL DEL NORTE

\begin{tabular}{ll}
\hline SCIAN & \\
\hline 112 & Cría y explotación de animales \\
114 & Pesca, caza y captura \\
115 & Servicios relacionados con las actividades agropecuarias y forestales \\
212 & Minería de minerales metálicos y no metálicos, excepto petróleo y gas \\
236 & Edificación \\
237 & Construcción de obras de ingeniería civil \\
238 & Trabajos especializados para la construcción \\
311 & Industria alimentaria \\
312 & Industria de las bebidas y del tabaco \\
313 & Fabricación de insumos textiles y acabado de textiles \\
314 & Fabricación de productos textiles, excepto prendas de vestir \\
315 & Fabricación de prendas de vestir \\
316 & Curtido y acabado de cuero y piel, y fabricación de productos de cuero, piel y \\
321 & materiales sucedáneos \\
322 & Industria de la madera \\
323 & Industria del papel \\
325 & Impresión e industrias conexas \\
326 & Industria química \\
327 & Industria del plástico y del hule \\
331 & Fabricación de productos a base de minerales no metálicos \\
332 & Industrias metálicas básicas \\
333 & Fabricación de productos metálicos \\
336 & Fabricación de maquinaria y equipo \\
337 & Fabricación de equipo de transporte \\
&
\end{tabular}


Otras industrias manufactureras

Comercio

Autotransporte de carga

Transporte terrestre de pasajeros, excepto por ferrocarril

Transporte turístico

Servicios relacionados con el transporte

Servicios de almacenamiento

Edición de periódicos, revistas, libros, software y otros materiales, y edición de estas publicaciones integrada con la impresión

Industria fílmica y del video, e industria del sonido

Radio y televisión

Otras telecomunicaciones

Instituciones de intermediación crediticia y financiera no bursátil

Actividades bursátiles, cambiarias y de inversión financiera

Compañías de fianzas, seguros y pensiones

Servicios inmobiliarios

Servicios de alquiler de bienes muebles

Servicios profesionales, científicos y técnicos

Servicios de apoyo a los negocios

Manejo de desechos y servicios de remediación

Servicios educativos

Servicios médicos de consulta externa y servicios relacionados

Hospitales

Residencias de asistencia social y para el cuidado de la salud

Otros servicios de asistencia social

Servicios artísticos, culturales y deportivos, y otros servicios relacionados

Museos, sitios históricos, zoológicos y similares

Servicios de entretenimiento en instalaciones recreativas y otros servicios recreativos

Servicios de alojamiento temporal

Servicios de preparación de alimentos y bebidas

Servicios de reparación y mantenimiento

Servicios personales

Asociaciones y organizaciones

Fuente: elaboración propia con base a INEGI (2013). 


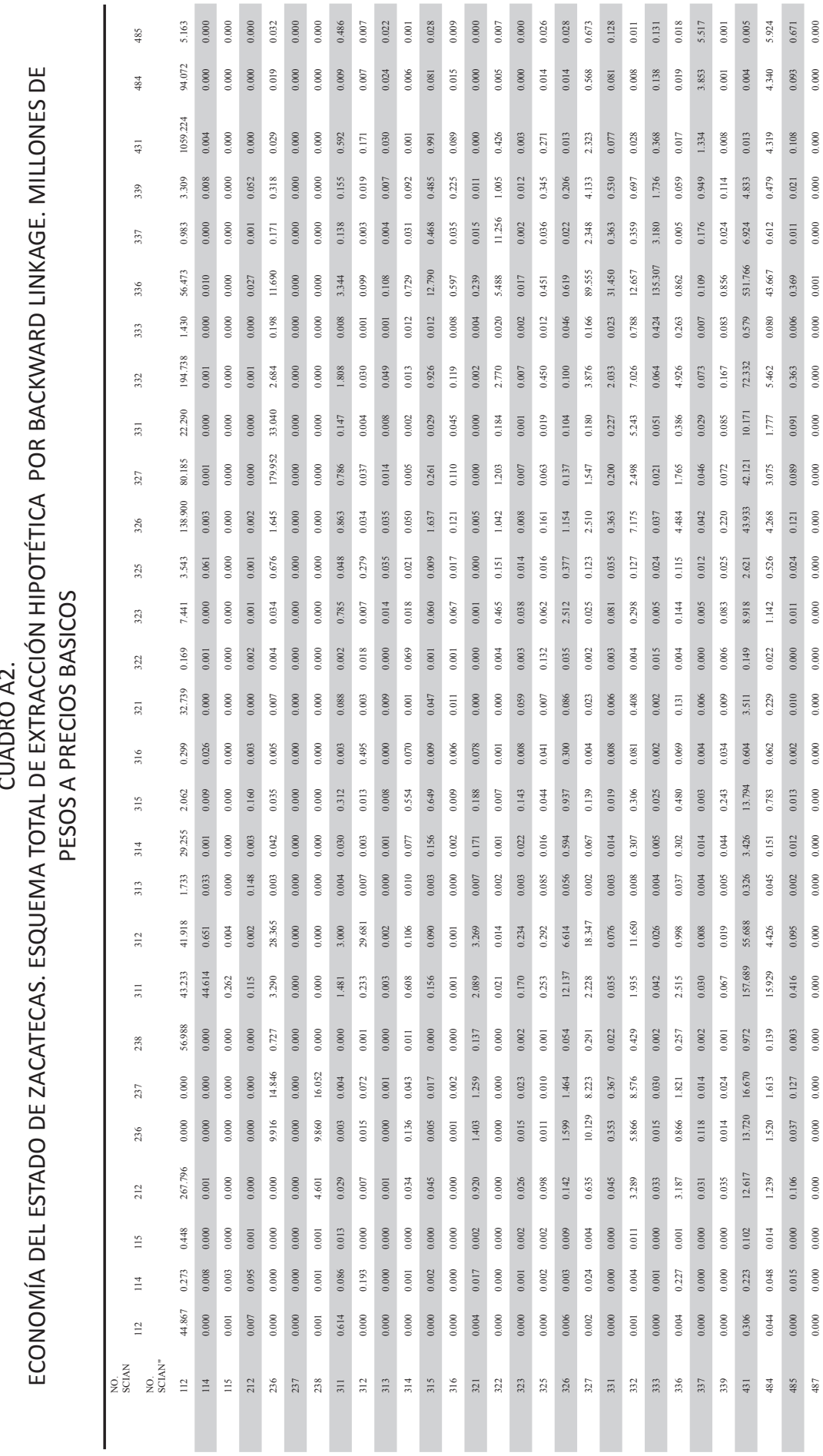




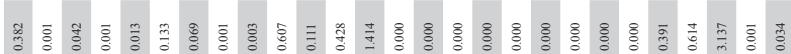

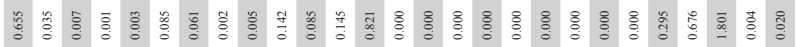

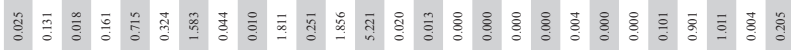

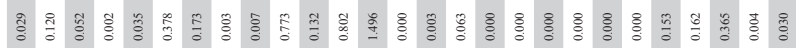

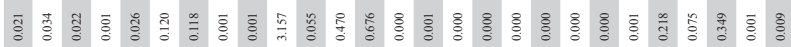

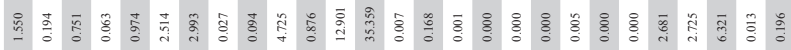

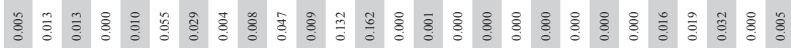

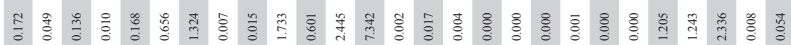

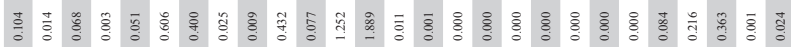

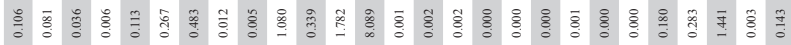

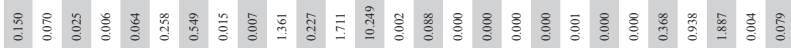

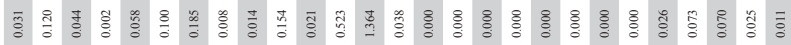

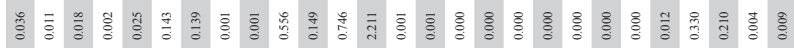

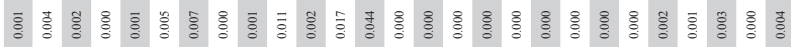

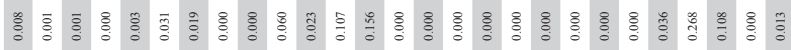

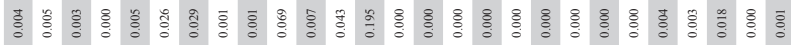

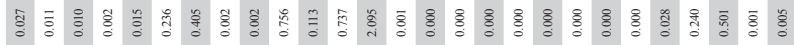

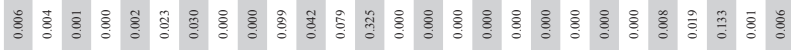

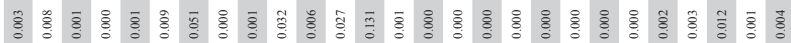

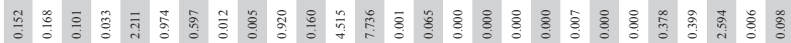

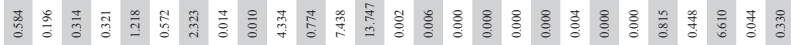

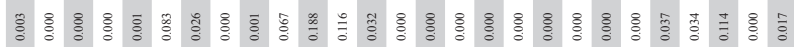

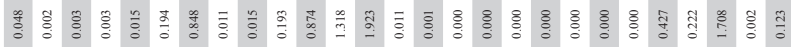

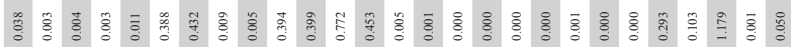

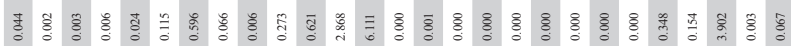

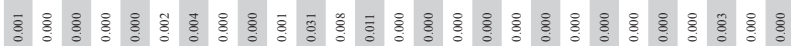

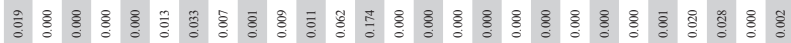

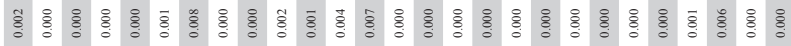

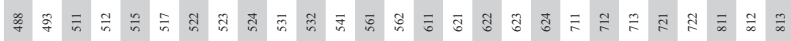




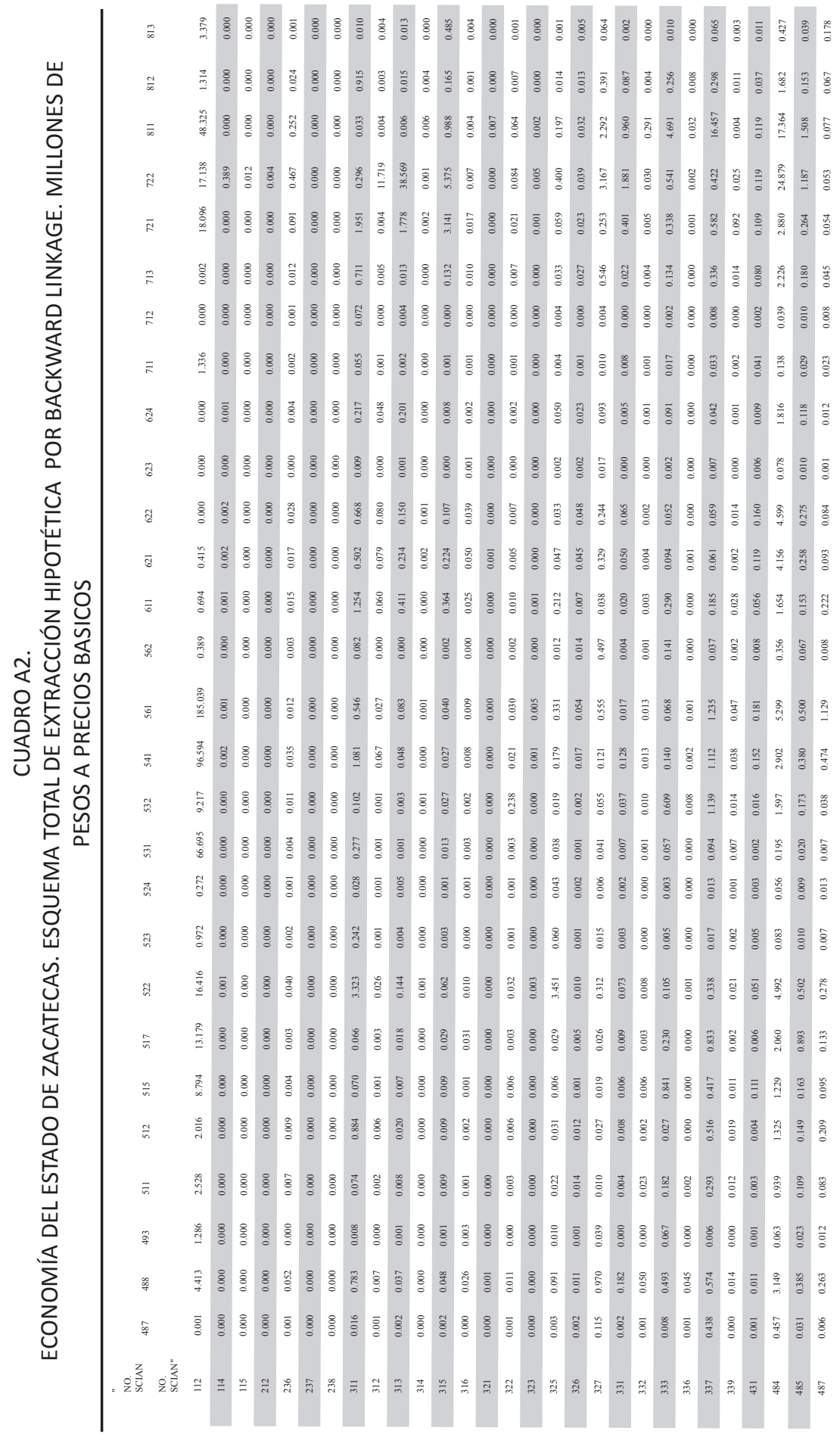




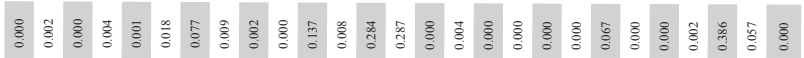

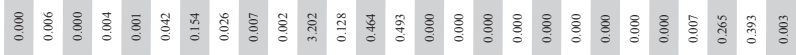

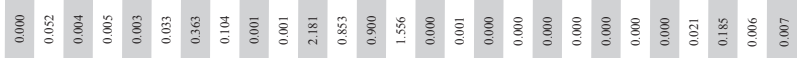

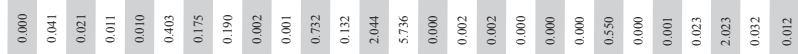

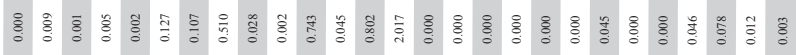

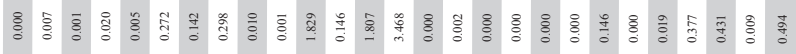

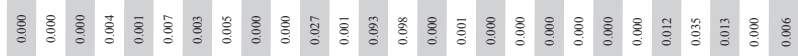

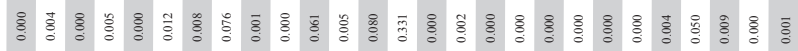

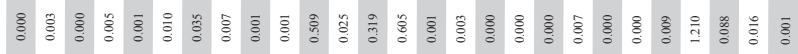

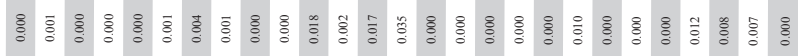

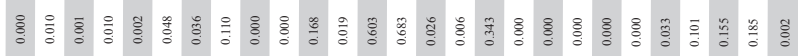

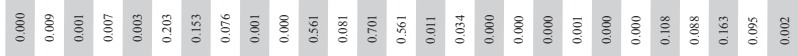

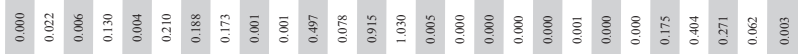

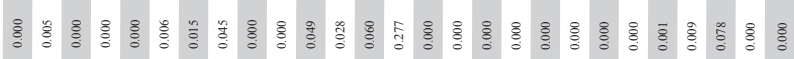

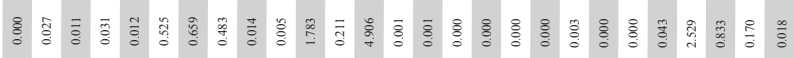

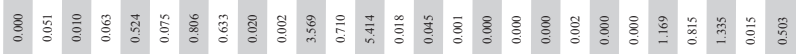

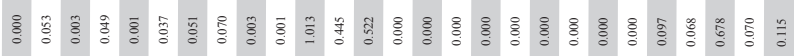

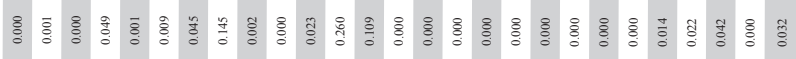

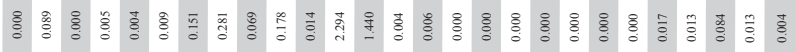

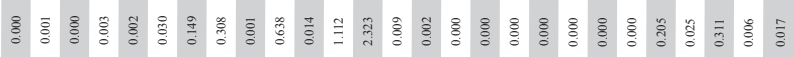

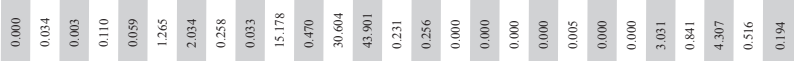

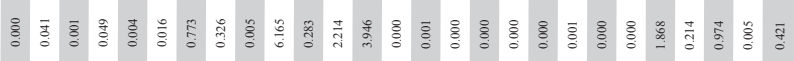

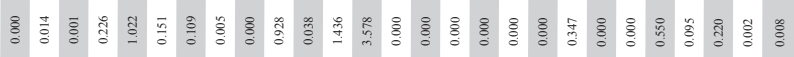

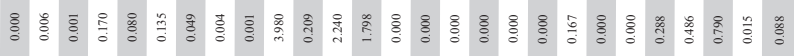

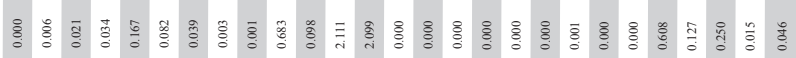

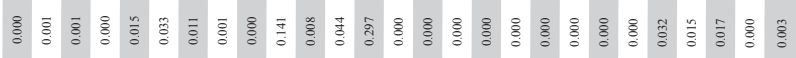

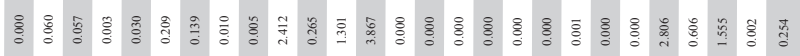

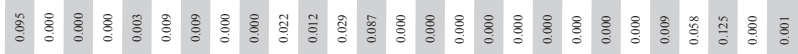

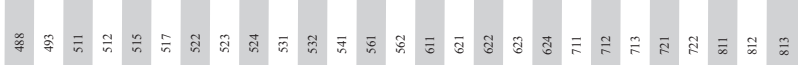


Paradigma económico 7 Año 12 Núm. 1

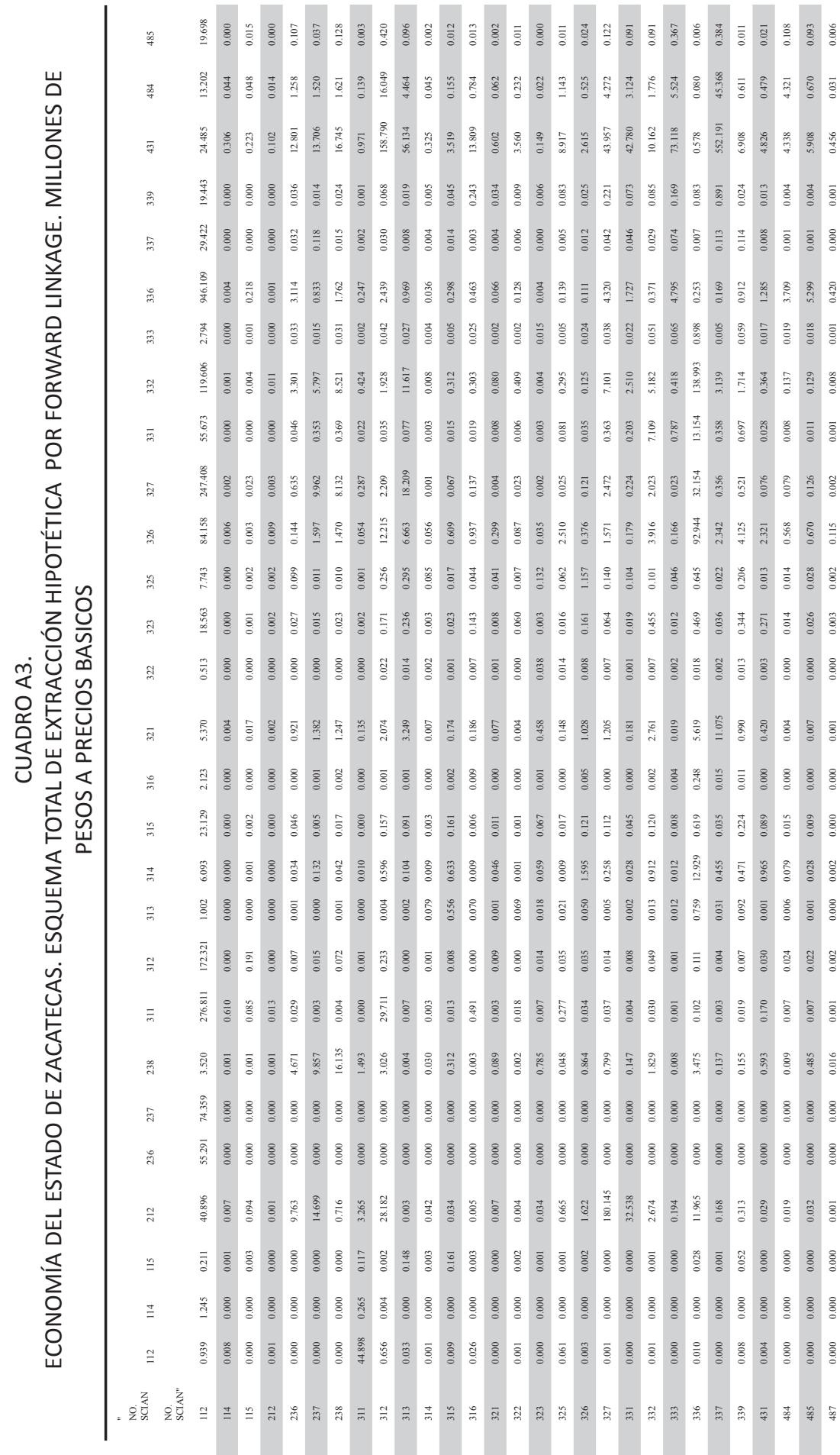




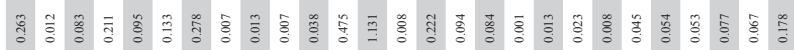

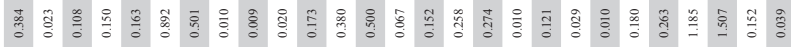

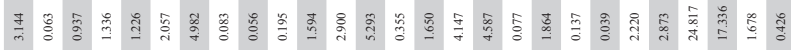

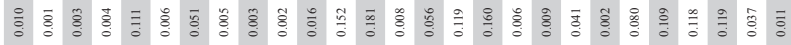

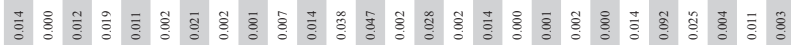

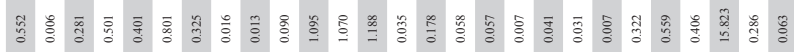

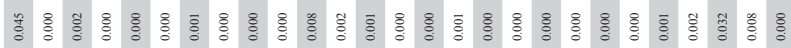

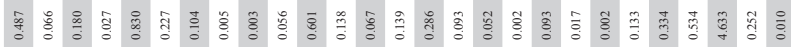

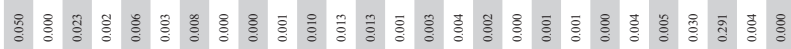

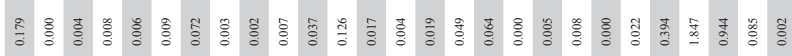

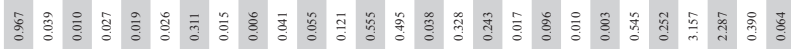

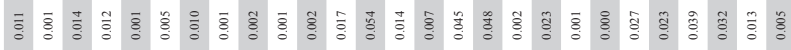

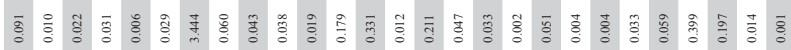

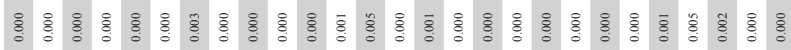

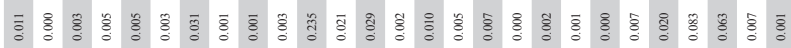

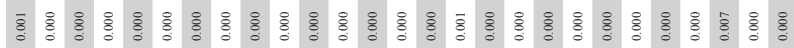

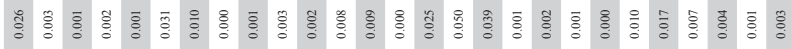
動 $\overline{\mathrm{g}}$

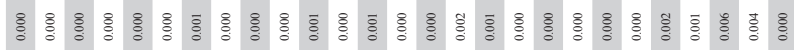

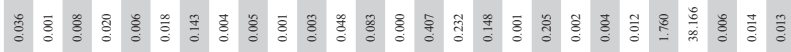

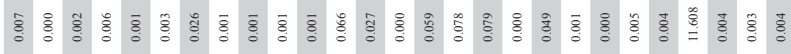

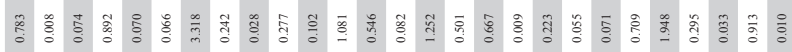

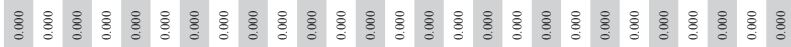

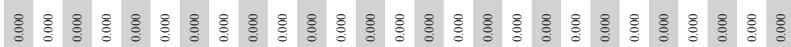
ఫ

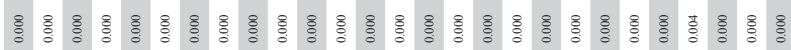

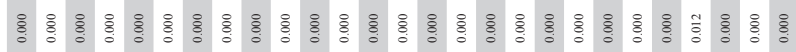

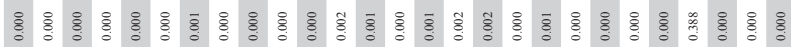

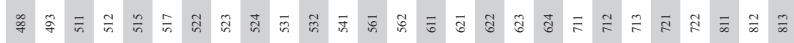




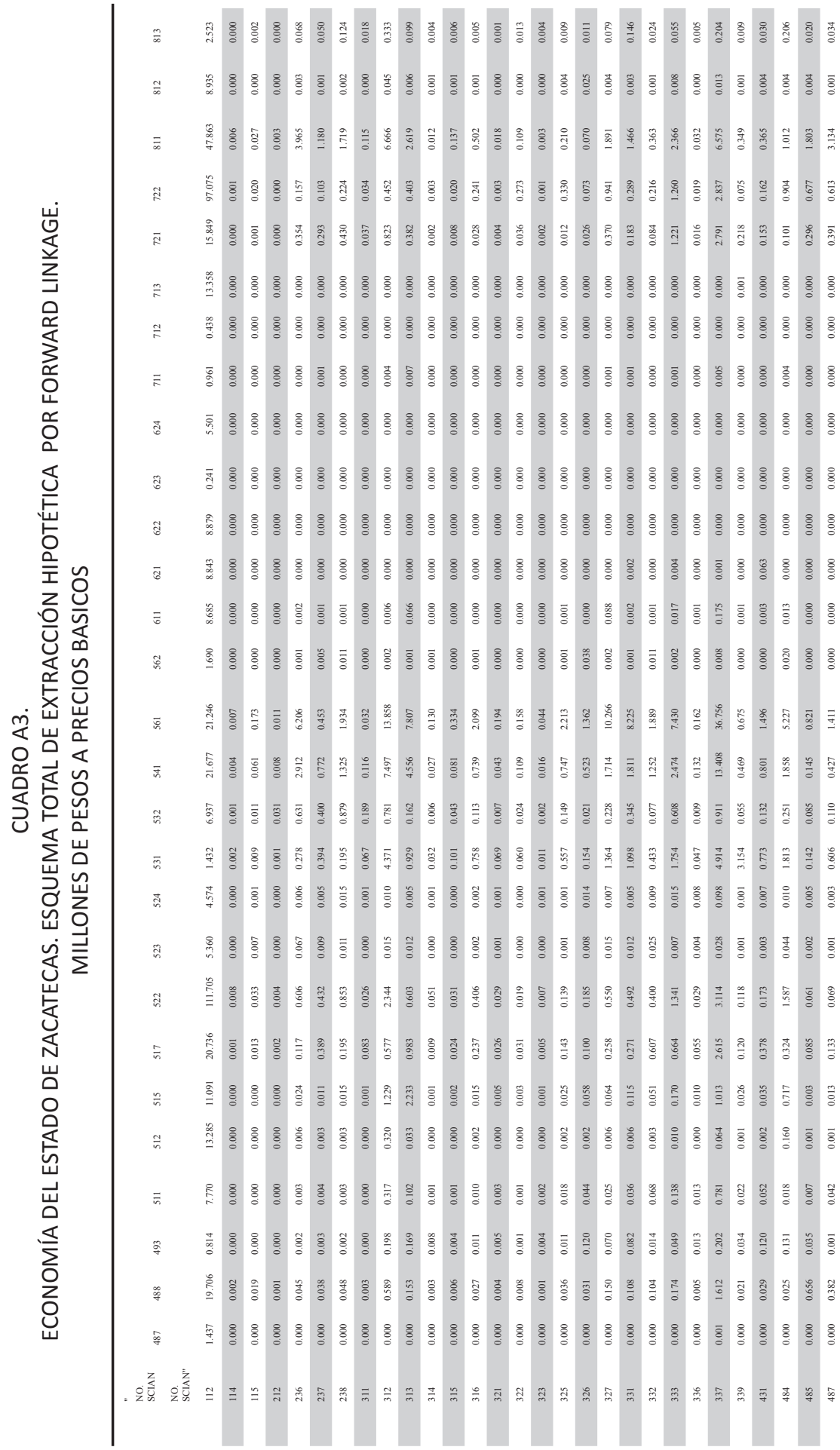




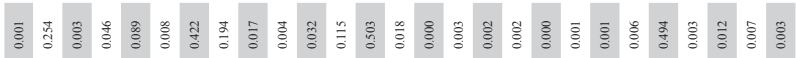

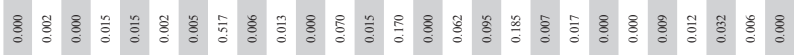

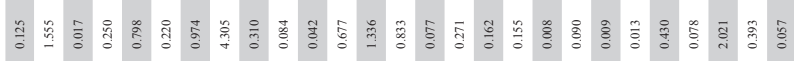

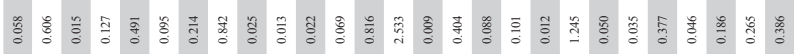

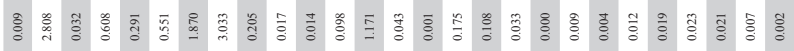

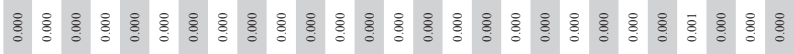

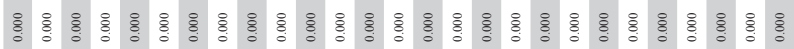

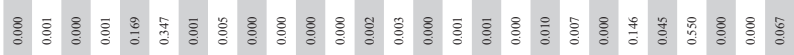

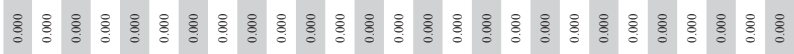

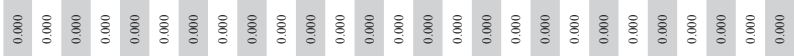

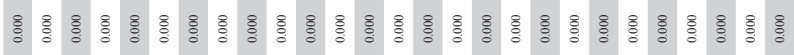

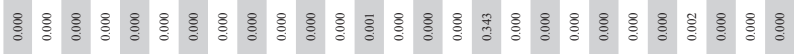

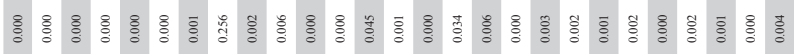

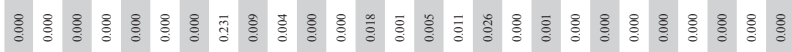

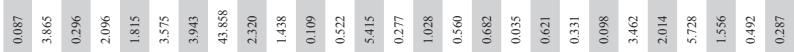

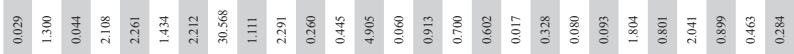

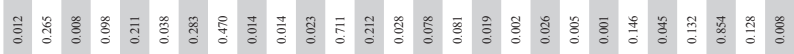

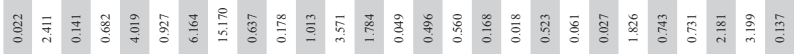

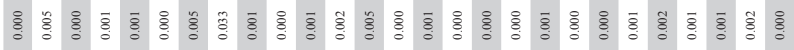

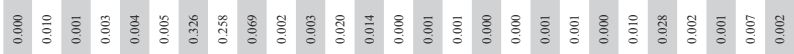

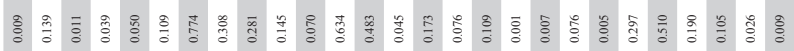

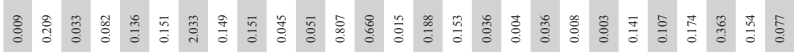

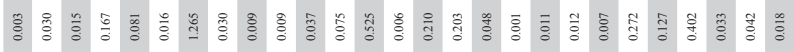

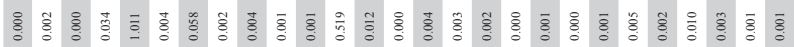

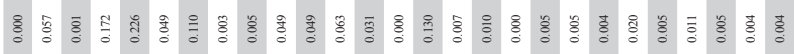

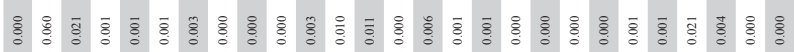

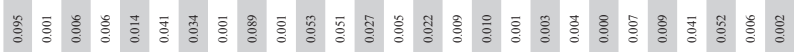

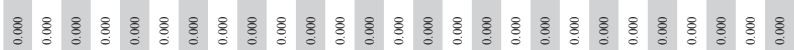

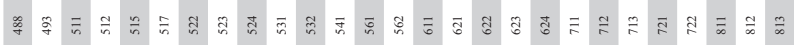

